\title{
Analysis of Destruction Process of the Primary Thrombus Under the Influence of the Blood Flow*
}

\author{
Koichiro YANO $^{* *}$, Daisuke MORI ${ }^{* * *}$, Ken-ichi TSUBOTA ${ }^{* * *}$, Takuji ISHIKAWA ${ }^{* * *}$, \\ Shigeo $\mathrm{WADA}^{* * * *}$ and Takami YAMAGUCHI ${ }^{* * *}$ \\ **Toyota Motor Corporation, \\ Toyota-cho, Toyota, Aichi 471-0826, Japan \\ ${ }^{\star * *}$ Dept. of Bioengineering and Robotics, Grad. Sch. Eng., Tohoku University, \\ 6-6-01 Aoba, Aoba-ku, Sendai, Miyagi 980-8579, Japan \\ E-mail: daisuke@pfs.mech.tohoku.ac.jp \\ ${ }^{* * * \star}$ Dept. of Mechanical Science and Bioengineering, Grad. Sch. Eng., Osaka University, \\ 1-3 Machikaneyama, Toyonaka, Osaka 560-8531, Japan
}

\begin{abstract}
Stokesian dynamics method based on the approximation of the additivity of velocities was employed to analyze the mechanical behavior of the primary thrombus under the blood flow. The mechanical interactions in the platelet-platelet aggregation and the platelet-surface adhesion via biological macromolecules such as von Willebrand factor and fibrinogen was modeled by the Voigt model. The process of the primary thrombus destruction was simulated under different levels of the mechanical interaction using the developed method. When the binding force was weak, which corresponds to the decline of the platelet function in the bleeding diseases, it was demonstrated that the platelets did not efficiently reside on the injured site of the vessel wall. The results show that our modeling can qualitatively demonstrate the effect of the mechanical interaction via adhesive macromolecules on the destruction process of the primary thrombus. Our modeling could be a powerful tool to better understand the physiological hemostatic mechanism as well as the pathology of the thrombosis and the bleeding disorders.
\end{abstract}

Key words: Stokesian Dynamics, Platelet, Plasma Proteins, Bleeding Diseases, Thrombosis

\section{Introduction}

Formation of thrombus, which is a coagulation formed on injured vascular lumen by blood cell constituents such as platelets and erythrocytes, is both physiologically and pathologically a crucial phenomenon. Physiologically, it plays an important role in hemostatic function. Under diseased conditions such as arteriosclerosis etc., on the other hand, the thrombus formation can be causes of fatal diseases, for example myocardial infraction, which block the blood supply to the periphery ${ }^{(1)}$. In contrast, failure of the thrombus formation due to platelet dysfunction, for instance Glanzmann thrombasthenia, von Willebrand disease, hemophilia, etc., shows a critical bleeding tendency ${ }^{(2)}$. Thus, better understanding the mechanism of the thrombus formation helps us clear not only the physiological hemostatic mechanism but also the pathology of the thrombosis and the bleeding disorders, which ultimately leading us to improve the treatment for such diseases 
including the development of the artificial platelet ${ }^{(3)}$.

Although the thrombus formation is regulated through a series of physiological processes, various mechanical factors are involved in the formation. In the platelet adhesion, platelets are recruited onto the injured surface of the vessel wall, whereas in the platelet aggregation, platelets cohere with each other. These phenomena are made up by combined forces between coagulation factors, which exist as long chain macromolecules in plasma, and the glycoprotein (GP) receptors on platelet ${ }^{(1,4-6)}$. Thus, the thrombus can be regarded as a mechanical structure constructed by the platelets which are built with physical interaction via the coagulation factors. Additionally, it is necessary to pay attention to that the thrombus is formed under fluid mechanical conditions due to the blood flow. Experimentally, it has been found that the number of platelets which are adhered onto collagen surface prepared to mimic the injured vessel wall changes depending on the magnitude of shear stress exerted on the surface ${ }^{(7,8)}$. Another example which shows the importance of fluid mechanical factor on the thrombus formation is the fact that the thrombus resists against the force exerted by the blood flow.

Even though recent powerful computational simulations have been attempted to clarify the mechanism of the process of the thrombus formation ${ }^{(9,10)}$, the effects of the mechanical factors have not been fully considered yet. The developed method in those studies did not concern with the mechanical aspect such as fluid-thrombus interactions and mechanical interactions involved in platelet adhesion and aggregation. More recently, our group conducted particle method to analyze not only the formation but also the collapse of primary thrombus in the blood flow, and demonstrated that the process of the thrombus formation was influenced by the relative magnitude of fluid mechanical force to the platelet-surface adhesive and platelet-platelet coherent forces ${ }^{(11)}$. In the study, however, the spatial resolution was not enough to express the hydrodynamic interactions between two close platelets.

In the present study, we employed Stokesian dynamics method based on the approximation of the additivity of velocities ${ }^{(12,13)}$ to analyze the mechanical behavior of the primary thrombus under the blood flow. Since the Stokesian dynamics method can consider more precisely the effect of multibody hydrodynamic interactions, it helps us to eliminate the disadvantage of the particle method used in the former study. For the mechanical interaction through the coagulation factors, Voigt model, which is composed of spring and dashpot, was introduced. We applied the developed method for the process of the primary thrombus destruction as the first trial. The computations were carried out for different levels of ability for the platelet adhesion and aggregation to study the effect of those on the destruction process.

\section{Methods}

\subsection{Stokesian dynamics method}

The framework of the method we employed followed Stokesian dynamics method which has been developed based on the approximation of the additivity of velocities and applied for ferromagnetic colloidal dispersions by Satoh et $\mathrm{al}^{(12,13)}$. In the present study, however, the binding force mediated by the coagulation factor was introduced instead of the magnetic force. The binding force was modeled using the Voigt model as described in the following section.

Stokesian dynamics is a powerful method to trace the motion of the multi-particles in suspension, and takes into account the hydrodynamic interactions between particles accurately. Although the approximation of the additivity of velocities is inferior to the other approximation of the additivity of forces ${ }^{(14)}$ in the reproduction of the lubrication effect, the calculation of the inverse matrix is unnecessary in the simulations ${ }^{(12,13)}$. Thus the additivity of velocities can considerably reduce the computational time, which means 
that it is applicable to a much larger system. Since we are interested in the thrombus formation, which is composed of a large amount of platelets, a larger system is desirable. Moreover, the lubrication effect is not thought to be significant in our system because the binding force is dominant in the case where the platelets close to each other. Also Stokesian dynamics based on the additivity of velocities is superior in the reduction of the computational time in comparison with the other computational fluid dynamics. Stokesian dynamics method does not require the spatial discretization for the dispersed medium, and employing the approximation of the additivity of velocities only needs $\mathrm{O}(3 N)$ operations to obtain three velocity components using Eq. (3), which will be subsequently derived. In contrast, other methods such as finite difference, finite element, lattice Boltzmann methods, etc. require a large amount of computational meshes for the particles and the dispersed medium, which enforce huge computational effort. Even a boundary element method, which only needs discretization for the surface of the object, may require 100-1000 surface meshes per one object and enforces $\mathrm{O}(3 N M)$ operations for tracing the motions of $N$ particles, where $M$ indicates the number of surface meshes per a particle.

The plasma, which is the dispersed medium in our system, is assumed to be incompressible Newtonian fluid. We consider the motion of platelets only near the vessel wall. Since the size of platelet is about $2 \mu \mathrm{m}$, we can assume that the flow field around platelets is governed by the Stokes equation

$$
\nabla p=\eta \nabla^{2} \boldsymbol{u},
$$

and the equation of continuity

$$
\nabla \cdot \boldsymbol{u}=0
$$

where $p$ is the pressure, $\eta$ is the viscosity, and $\boldsymbol{u}$ is the velocity vector.

The other cell constituents than platelet were neglected, because there is a plasma layer in the vicinity of vessel walls, where large blood cells do not exist. The platelets were idealized as solid sphere particles. Although this is a strong simplification, activated platelets have rather spherical shape than disc shape ${ }^{(15)}$. Moreover, for the artificial platelets, various kinds of microspheres can be used ${ }^{(3)}$. Although activated platelets possess extensive pseudopodia ${ }^{(15)}$, there is no accepted model which appropriately deals with such objects with complex shape. In this study, we only deal with activated platelets, and they are modeled as solid spheres with near field forces. We consider the dispersions of multiple spheres with identical radius. Stokesian dynamics based on the additivity of velocities due to the force exerted on the particle yields the particle velocity of:

$$
\boldsymbol{v}_{\alpha}=\boldsymbol{U}\left(\boldsymbol{r}_{\alpha}\right)+\frac{1}{\eta}\left\{\frac{1}{6 \pi a} \boldsymbol{F}_{\alpha}+\sum_{\beta=1(\neq \alpha)}^{N}\left(\boldsymbol{a}_{\alpha \alpha}-\frac{1}{6 \pi a} \boldsymbol{I}\right) \cdot \boldsymbol{F}_{\alpha}+\sum_{\beta=1(\neq \alpha)}^{N} \boldsymbol{a}_{\alpha \beta} \cdot \boldsymbol{F}_{\beta}\right\}+\sum_{\beta=1(\neq \alpha)}^{N} \tilde{\boldsymbol{g}}_{\alpha}: \boldsymbol{E},
$$

where $\boldsymbol{U}\left(\boldsymbol{r}_{\alpha}\right)$ is the velocity of background flow filed at the position of particle $\alpha, \boldsymbol{F}_{i}(i=\alpha$, $\beta$ ) are the forces acting on the particle $i, a$ is the particle radius, $\boldsymbol{E}$ is the rate-of-strain tensor, $\boldsymbol{I}$ is the unit tensor, $\boldsymbol{a}_{i j}$ and $\tilde{\boldsymbol{g}}_{i}$ are the mobility tensors, and $N$ indicates the number of the particles in the system ${ }^{(12,13)}$. The mobility tensors can be found in a standard text, such as Kim and Karrila ${ }^{(16)}$. In the present study, $\boldsymbol{F}_{i}$ is considered as a sum of the binding force induced between the particles by the coagulation factor, $\boldsymbol{F}_{i}^{(b)}$, and the contact force induced by the collision of particles, $\boldsymbol{F}_{i}^{(c)}$, thus,

$$
\boldsymbol{F}_{i}=\boldsymbol{F}_{i}^{(b)}+\boldsymbol{F}_{i}^{(c)} \text {. }
$$

Since the platelets are too large for Brownian motion to be taken into account, it does not appear in Eq. (3). We neglected the difference in the density between the plasma and the platelet as well. The rotational motion of particles was neglected in this study. Because the rotational velocity at $\boldsymbol{r}$ due to the torque exerted on a particle at the origin of coordinate decays as $r^{-2}$, where $r$ is the distance, in the far field. On the other hand, the velocity at $r$ due to the force exerted on a particle at the origin of coordinate decays as $r^{-1}$, which is much larger in the far field. The update for the particle position was done using a modified Euler method given by: 


$$
\left[\boldsymbol{r}_{\alpha}\right]_{t+\Delta t}=\left[\boldsymbol{r}_{\alpha}\right]_{t}+\Delta t\left(\left[\boldsymbol{v}_{\alpha}\right]_{t}+\left[\boldsymbol{v}_{\alpha}\right]_{t-\Delta t}\right) / 2
$$

where the subscript $t, t-\Delta t$, or $t+\Delta t$ indicates that the value is for current, previous, or next time step, respectively.

\subsection{Binding force via plasma proteins}

In order to express the binding force between particles via the coagulation factor, we introduce the Voigt model which consists of parallel spring and dashpot (Fig. 1 (a)). The modeling was based on the idea proposed to model material such as mortars which mediate the interaction between sands and pebbles in concrete structures by Hakuno et $\mathrm{al}^{(17)}$. Von Willebrand factor (vWF) and fibrinogen (Fbg) are well-known plasma proteins which play an important role to form the primary thrombus. These proteins exist in form of long chain macromolecules in the plasma. For example, vWF forms the multimer which can have various molecular weights from 500,000 to $2,000,000 \mathrm{M}$. The structure is filamentous with a diameter 2-3 nm and a length of up to $1,300 \mathrm{~nm}$, approaching the dimension of a platelet diameter, as well as loosely coiled molecules with an apparent diameter of $200-300 \mathrm{~nm}^{(6)}$. It has been known that the size is well functionally regulated under the fluid mechanical factor such as the shear stress. In order to reproduce such function as long chain molecules, we define separation vector $\boldsymbol{s}$ as

$$
\boldsymbol{s}=\frac{\boldsymbol{r}_{j}-\boldsymbol{r}_{i}}{\left|\boldsymbol{r}_{j}-\boldsymbol{r}_{i}\right|}\left\{\left|\boldsymbol{r}_{j}-\boldsymbol{r}_{i}\right|-2 a\right\},
$$

and exert the binding force as follows. We assume that the binding force is generated only when two particles, which once satisfied $|\boldsymbol{s}| \leq L_{0}$, goes away from each other so that $\boldsymbol{s}$ satisfies $L_{0} \leq|\boldsymbol{s}| \leq \varepsilon L_{0}$, where $L_{0}$ is a natural length for the spring. The spring is set to be broken once $|\boldsymbol{s}|$ exceeds $\varepsilon L_{0}$ and resist only against the stretch, where $\varepsilon$ is the maximum stretch of the spring. By assuming Voigt model as shown in Fig. 1(a), the binding force can be given as

$$
\left.\boldsymbol{F}_{i j}^{(b)}=\frac{\boldsymbol{s}}{|\boldsymbol{s}|} \mid K^{(b)}\left\{|\boldsymbol{s}|-L_{0}\right\}+\eta^{(b)} \frac{|\Delta \boldsymbol{s}|}{\Delta t}\right\rfloor
$$

where $K^{(b)}$ and $\eta^{(b)}$ are the spring elastic modulus and the damper viscous coefficient, respectively, and $\Delta t$ is a time interval.

Although the physiological process of the platelet adhesion to the injured surface is different from that of the platelet coagulation, the main factors which bridge between the platelet and the wall are vWF and Fbg similarly as the platelet aggregation. Hence, the binding force between the platelet and the wall, $\boldsymbol{F}_{\text {wall }}^{(b)}$, was modeled using the Voigt model too, as shown in Fig. 1 (b). Eventually, the binding force acting on a particle $i$ is expressed as

$$
\boldsymbol{F}_{i}^{(b)}=\sum_{j} \boldsymbol{F}_{i j}^{(b)}+\boldsymbol{F}_{\text {wall }}^{(b)}
$$

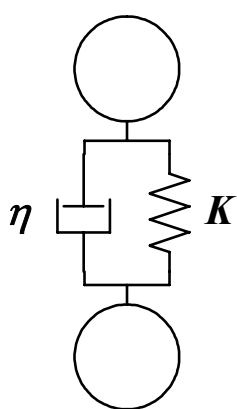

(a)

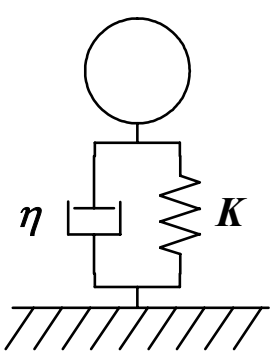

(b)

Fig. 1 Voigt model used for the binding force and the contact force. The Voigt model consists of parallel spring, which has elastic modulus $\eta$, and dashpot, which has viscosity coefficient $K$. The identical model was applied for both (a) the particle-particle and (b) the particle-surface interactions. 


\subsection{Contact force}

Essentially, the lubrication effect caused between nearly touching particles induces very large hydrodynamic force, which avoids the direct collision of the particles. Stokesian dynamics has ability to reproduce the lubrication effect. However, in our system, such rigorous treatment is not so important, because the balance of the binding force and viscous drag force dominates the destruction process of the platelets. The force was modeled using the Voigt model similarly as the binding force. But the contact force is induced so that the spring can resist against the compression, only when the particles satisfy the following equation:

$$
\left|\boldsymbol{r}_{j}-\boldsymbol{r}_{i}\right|-2 a \leq 0 .
$$

The force is expressed as:

$$
\left.\boldsymbol{F}_{i j}^{(c)}=\frac{\boldsymbol{s}}{|\boldsymbol{s}|}\left|K^{(c)}\right| \boldsymbol{s} \mid+\eta^{(c)} \frac{|\Delta \boldsymbol{s}|}{\Delta t}\right]
$$

where $K^{(c)}$ and $\eta^{(c)}$ are the spring elastic modulus and the damper viscous coefficient, respectively.

Similarly, the contact force between the platelet and the wall, $\boldsymbol{F}_{\text {wall }}^{(c)}$, is modeled. Thus, the contact force acting on a particle $i$ is expressed as

$$
\boldsymbol{F}_{i}^{(c)}=\sum_{j} \boldsymbol{F}_{i j}^{(c)}+\boldsymbol{F}_{\text {wall }}^{(c)} \text {. }
$$

\subsection{Parameter for reproducing dysfunction of coagulation factor}

Qualitative or quantitative dysfunction of coagulation factors results in the failure of the thrombus formation. One of examples of such dysfunction is von Willebrand disease, which shows the dysfunction of vWF, and the other is Glanzmann thrombasthenia, which shows the dysfunction of GP IIb/IIIa, a receptor for vWF and Fbg. These dysfunctions result in decrease of the coagulation factors which have ability to bridge between the particles. In order to model that, we set a parameter $N_{\text {bind }}$, which shows the number of the platelets with which one particle can interact. The smaller $N_{\text {bind }}$ reproduces the higher level of dysfunction. Introducing the parameter, $N_{\text {bind }}$, rather than controlling adhesive force comes from the fact that the receptors are not uniformly distributed on the activated platelet. For instance, the population of the receptor GP Ib $\alpha$ was found to be coarse on the pseudopodia, and partly clustered on the activated platelet ${ }^{(18)}$. We assumed that such uneven distribution of the receptors does not necessarily induce the reduction of the adhesive force.

\subsection{Two dimensional thrombus destruction model}

We applied the developed method for the process of the primary thrombus destruction as the first trial (Fig. 2). All the particles have an identical radius $a=1.0[\mu \mathrm{m}]$. We considered the interactions between particles and the wall only in $x=0-200[\mu \mathrm{m}]$ region, and the injured wall was set between $x=10-50[\mu \mathrm{m}]$. The particles were initially arranged above the region in the shape of Gauss distribution to mimic the primary thrombus. The number of the particles was $N=105$. In this study, we neglected the effect of the roughness of the endothelial surface on the flow, because the height of the endothelial cell is significantly small compared with the size of the thrombus with the height of approximately $20 \mu \mathrm{m}$. There were no other particles than the initial ones in the domain. The background flow field was assumed to be Couette flow which is expressed as $\boldsymbol{U}(\boldsymbol{r})=\dot{\gamma} y \boldsymbol{\delta}_{x}$ with $\dot{\gamma}=5000\left[\mathrm{~s}^{-1}\right]$, where background flow means the induced flow when there is no particles. For this case, the rate-of-strain tensor $\boldsymbol{E}$ has only $E_{x y}$ and $E_{y x}$ as nonzero components; $E_{x y}=E_{y x}=\dot{\gamma} / 2$. For the viscosity of the disperse medium, that for water was used: $\mu=0.797 \times 10^{-3}$ [Pa.s]. For the properties of the Voigt model applied for the contact force, those of glass bead are used; $K^{(c)}=4.9 \times 10^{9}[\mathrm{~Pa}]$, and $\mu^{(c)}=1.43 \times 10^{-3}$ [Pa.s]. Since the properties for the coagulation factors such as vWF or Fbg has been 
hardly known, we attempted supplemental simulations and set $K^{(b)}=4.0 \times 10^{2}[\mathrm{~Pa}]$, and $\mu^{(b)}=$ $4.08 \times 10^{-6}[\mathrm{~Pa} \cdot \mathrm{s}]$. The natural length of the spring was assumed to be quarter of the particle diameter; $L_{0}=0.5[\mu \mathrm{m}]$. The maximum stretch of the spring $\varepsilon$ was set to be 1.05 . Table 1 summarizes all the parameters. The computations are carried out for three different $N_{\text {bind }}$ of 0,5 , and 10 . The time increment $\Delta t$ was set to be $1.0 \times 10^{-9}$ [s], and the computations were carried out for $t=2.0[\mathrm{~s}]$ with $2 \times 10^{9}$ timesteps.

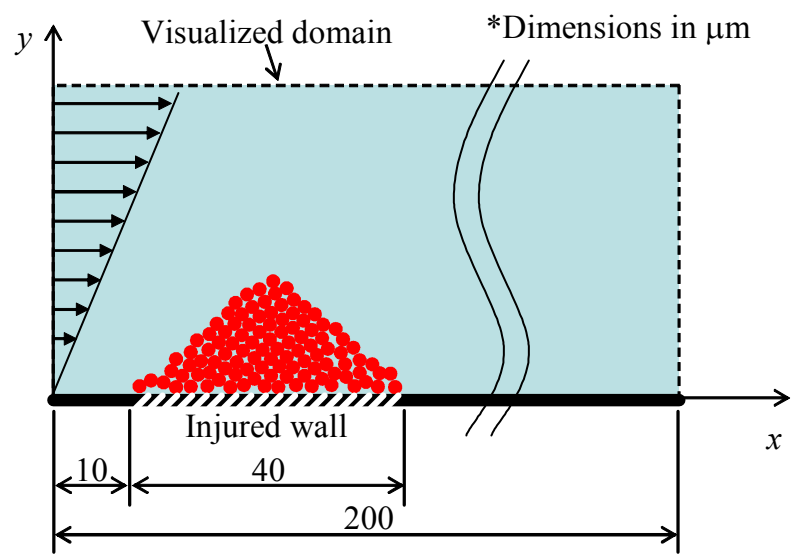

Fig. 2 Monolayer model for the destruction process of the primary thrombus. Couette flow was given for the background flow field. The surface, where the contact force was estimated, was set on $y=0$. The injured site, where the binding force was estimated, was set on the region $x=10-50[\mu \mathrm{m}]$. Particles were piled up above the injured site in shape of Gaussian distribution, simulating the primary thrombus.

Table 1 Parameters used for the background flow field, the disperse medium, the particle, the contact force model, and the adhesive force model.

\begin{tabular}{lll}
\hline Background flow field & Shear rate & $\dot{\gamma}=5000\left[\mathrm{~s}^{-1}\right]$ \\
\hline Disperse medium & Viscosity & $\mu=0.797 \times 10^{-3}[\mathrm{~Pa} \cdot \mathrm{s}]$ \\
\hline Particle & Radius & $a=1.0[\mu \mathrm{m}]$ \\
& Total number & $N=105$ \\
\hline Contact force model & Elastic modulus & $K^{(c)}=4.9 \times 10^{9}[\mathrm{~Pa}]$ \\
& Viscous coefficient & $\mu^{(c)}=1.43 \times 10^{-3}[\mathrm{~Pa} \cdot \mathrm{s}]$ \\
\hline Adhesive force model & Elastic modulus & $K^{(b)}=4.0 \times 10^{2}[\mathrm{~Pa}]$ \\
& Viscous coefficient & $\mu^{(b)}=4.08 \times 10^{-6}[\mathrm{~Pa} \cdot \mathrm{s}]$ \\
& Natural length & $L_{0}=0.5[\mu \mathrm{m}]$ \\
& Maximum stretch & $\varepsilon=1.05$ \\
\hline
\end{tabular}

\section{Results and Discussion}

Three different cases for $N_{\text {bind }}$ of 0,5 , and 10 were simulated under the set of the parameters shown in Table 1. Although it is unnatural that the thrombus is formed in the case of $N_{\text {bind }}=0$, in which no adhesive force is induced between platelets, we analyzed such extreme case for the comparison with the other cases of $N_{\text {bind }}=5$, and 10 . The time series of the motion of the particles are visualized in Figs 3-5. It was observed that the original thrombus gradually collapsed according to the shear flow in all the cases. However, the manner of the collapse was changed depending on $N_{\text {bind }}$. In the case of $N_{\text {bind }}$ 
$=0$, since the adhesive force was not induced between the particles, the particles were separated individually from the original primary thrombus (Fig. 3). In contrast, in the cases of $N_{b i n d}=5$, and 10, it is found that the particles separated from the original primary thrombus tend to form aggregations due to the adhesive force caused between the particles (Figs 4, and 5). In those two cases, the particles resisted against the fluid mechanical force to reside longer around the region of the original thrombus compared with the case of $N_{\text {bind }}$ $=0$. It resulted in shorter movement of the particles.

Figure 6 shows the time course of the number of colonies. One colony was defined as a cluster in which the gap between neighboring particles $|\boldsymbol{s}|$ satisfied $|\boldsymbol{s}| \leq \varepsilon L_{0}$. Thus, the original primary thrombus was regarded as one colony. The number of colonies was counted at each instant. In the case of $N_{\text {bind }}=0$, the number of colonies tends to increase with respect to the time, which resulted from that the particles were individually separated from the original thrombus. In the cases of $N_{\text {bind }}=5$, and 10, clustered particles were separated from the original thrombus so that the number of colonies much slowly changed as compared to the case of $N_{\text {bind }}=0$.

Figure 7 shows the time course of the number of platelets which resided above the region of $x=10-50[\mu \mathrm{m}]$, where the injured surface was assumed. It is seen that the greater $N_{\text {bind }}$ is, the larger amount of particles resides on the injured site for longer time. For example, at $t=0.6[\mathrm{~s}]$, the counts of the particles for each case are 54, 56, and 64 for $N_{\text {bind }}=0,5$, and 10, respectively. After that, the significant difference in the particle counts between $N_{\text {bind }}=0$, and 5 become to disappear. In the case of $N_{\text {bind }}=10$, on the other hand, larger amount of particles still stay on the injured region compared with the other two cases. For example, at $t=1.6$ [s], the particle count is 34 for $N_{\text {bind }}=10$ against 28 for $N_{\text {bind }}$ $=0$, and 5. Since small value of $N_{\text {bind }}$ can simulate the decline of the platelet function in the bleeding diseases such as von Willebrand disease, those results are thought to express the degradation of hemostatic function due to the dysfunction of platelets.

In the present study, we proposed a method to analyze the mechanical behavior of the primary thrombus under the blood flow by adopting the Sokesian dynamics method, which considers precisely the effect of multibody hydrodynamics interactions. Biological macromolecules such as vWF and Fbg, which mediate mechanical interactions between the platelets, were modeled by the Voigt model. One of the advantages of the proposed method is that the modeling is naturally based on the physiological nature of the discrete objects of the platelets. As is encountered in the various fields of the hemorheological study, it is very difficult task to overcome how to model the blood as continuum while it is composed of a great number of cellular components with a very wide variety of configuration as well as physical and physiological nature. Moreover, the discrete method in general, especially owing to the great advancement of computing technologies, is undoubtedly most potential way to analyze such inherently discrete phenomena. Another advantage of the method is that the near field interaction via biological macromolecules can be simply modeled in the way that their own physical and physiological nature is expressed. Thus the computational load to express the complex physics in the near field interaction can be considerably reduced.

We could qualitatively demonstrate the effect of the coagulation factors such as vWF and Fbg on the destruction process of the primary thrombus using our proposed method. In the simulations considering high level of binding ability between the particles and between the particle and the injured wall (i.e. $N_{\text {bind }}=5$, and 10 ), the results demonstrated that larger amount of particles can reside on the injured wall for longer time. For the normal physiological hemostatic function, the primary thrombus formed on the injured site must stay there for some duration of time until the following secondary thrombus formation 

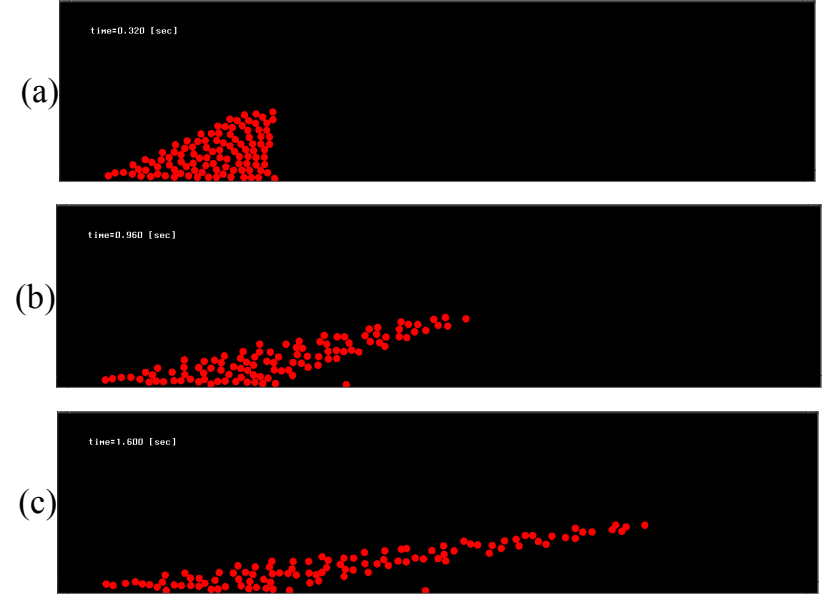

Fig. 3 The destruction process of the primary thrombus obtained from the computation for $N_{b i n d}=0$. (a) $t$ $=0.32[\mathrm{~s}] . \quad$ (b) $t=0.96[\mathrm{~s}] . \quad$ (c) $t=1.60[\mathrm{~s}]$. Since the adhesive force was not induced between the particles in this case, the particles were separated individually from the original primary thrombus
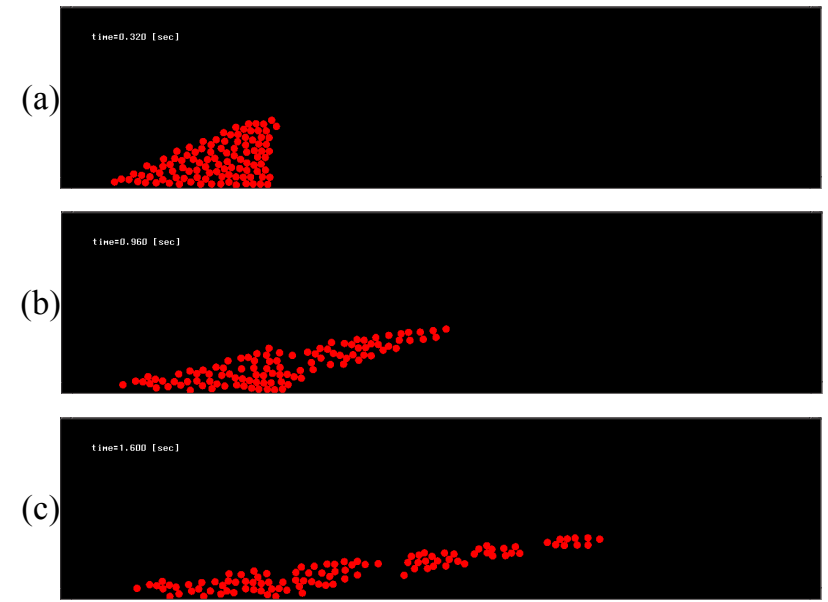

Fig. 4 The destruction process of the primary thrombus obtained from the computation for $N_{\text {bind }}=5$. (a) $t$ $=0.32[\mathrm{~s}] . \quad$ (b) $t=0.96[\mathrm{~s}] . \quad$ (c) $t=1.60[\mathrm{~s}] . \quad$ The particles separated from the original primary thrombus tend to form aggregations due to the adhesive force caused between the particles.
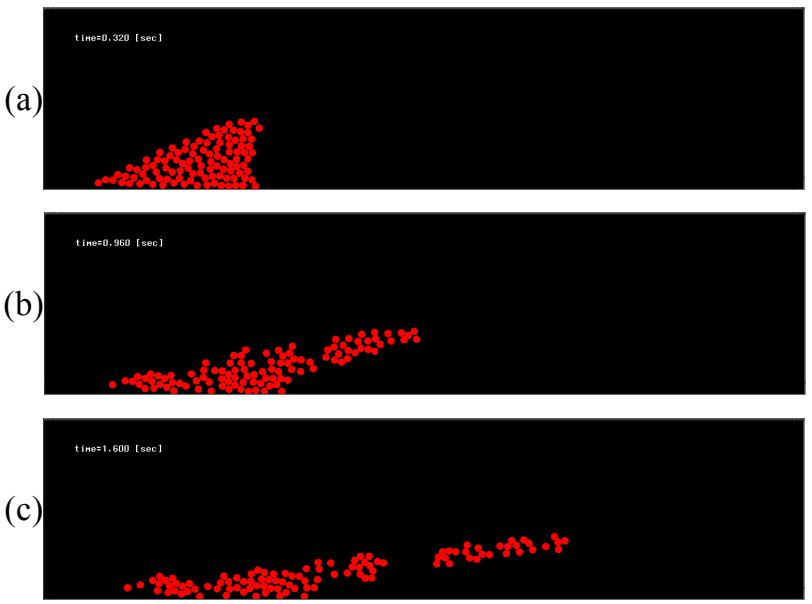

Fig. 5 The destruction process of the primary thrombus obtained from the computation for $N_{b i n d}=10$. (a) $t=0.32[\mathrm{~s}] . \quad$ (b) $t=0.96[\mathrm{~s}] . \quad$ (c) $t=1.60$ [s]. Similarly for the case of $N_{b i n d}=5$, the particles separated from the original primary thrombus tend to form aggregations due to the adhesive force. The particles resisted against the fluid mechanical force to reside longer around the region of the original thrombus compared with the other cases. It resulted in shorter movement of the particles. 
is initiated. Our proposed method may play a role to predict what critical magnitude of the mechanical interaction mediated by coagulation factors results in the stable primary thrombus or in the collapse. Such information may contribute to better understanding of the physiological hemostatic mechanism as well as the pathology of the thrombosis and the bleeding disorders, which ultimately leading us to improve the treatment of the diseases.

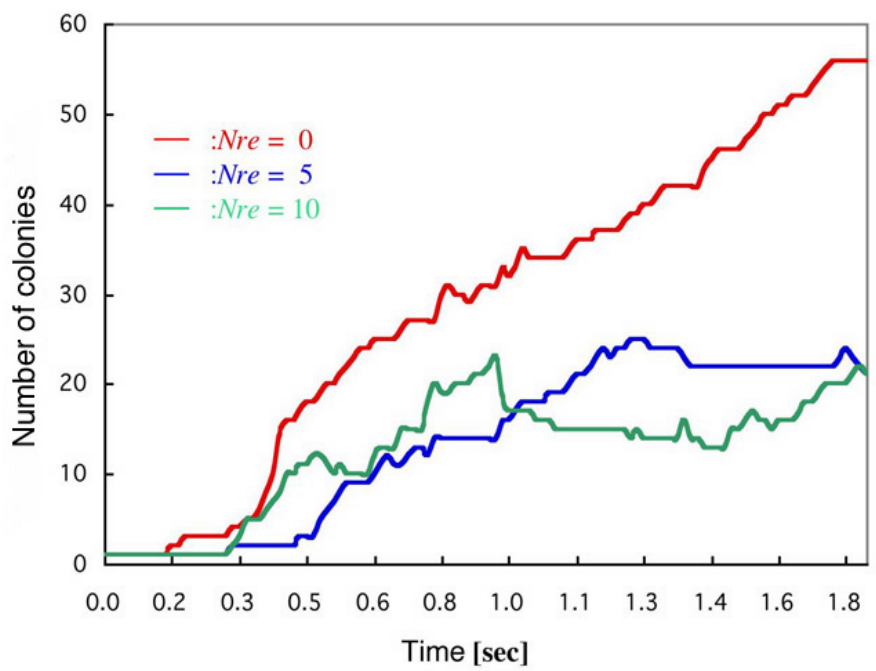

Fig. 6 Time course of number of colonies. One colony was defined as a cluster in which the gap between neighboring particles $|\boldsymbol{s}|$ satisfied $|\boldsymbol{s}| \leq \varepsilon L_{0}$. In the case of $N_{\text {bind }}=0$, the number of colonies tends to increase with respect to the time. In the cases of $N_{\text {bind }}=5$, and 10 , the number of colonies much slowly changed as compared to the case of $N_{\text {bind }}=0$.

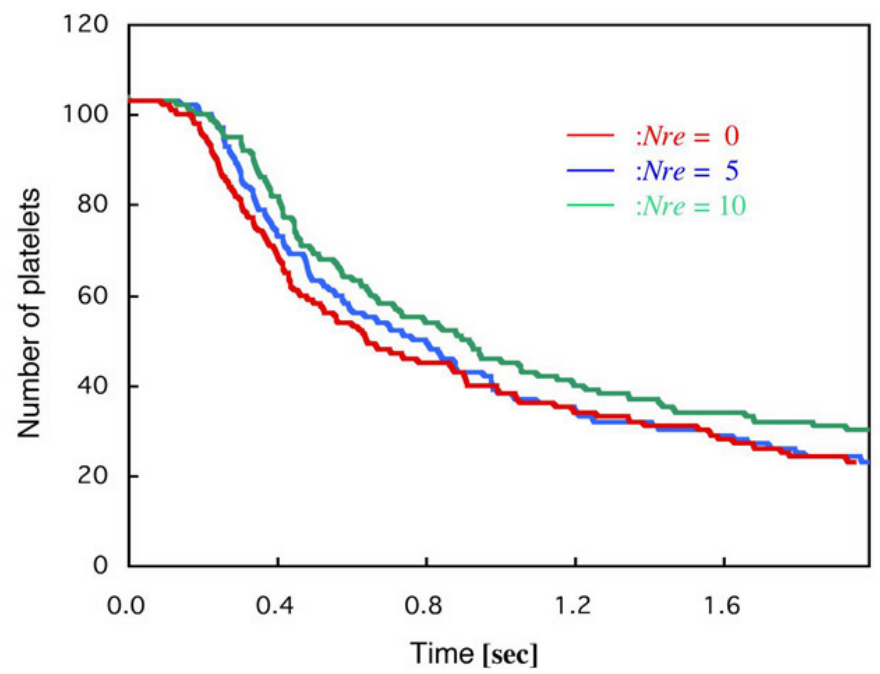

Fig. 7 Time course of the number of platelets which resided above the region of $x=10-50[\mu \mathrm{m}]$, where the injured surface was assumed. The significant difference in the particle counts between $N_{\text {bind }}=0$, and 5 eventually became to disappear. In the case of $N_{\text {bind }}=10$, on the other hand, larger amount of particles stayed on the injured region compared with the other two cases.

\section{Conclusion}

The mechanical behavior of the primary thrombus under the blood flow has been modeled by adopting the Sokesian dynamics method and modeling the mechanical interaction via biological macromolecules such as vWF and Fbg by the Voigt model. The proposed method was applied for the collapse process of the primary thrombus formation, where the three different levels of the binding ability were considered. Results show that 
our modeling can qualitatively demonstrate the effect of the mechanical interaction via adhesive macromolecules on the destruction process of the primary thrombus. It was demonstrated that when the binding force is weak, which can corresponds to the decline of the platelet function in the bleeding diseases, the platelets can not efficiently reside on the injured site of the vessel wall. Our modeling could be a powerful tool to better understand the physiological hemostatic mechanism as well as the pathology of the thrombosis and the bleeding disorders. For instance, that may play a role to predict what critical magnitude of the mechanical interaction in the platelet-platelet aggregation and the platelet-surface adhesion results in the stable primary thrombus or in the collapse. Such information would be useful to improve the treatment of the diseases including the development of the artificial platelet. In future, the simulations will be extended to cover not only the collapse process but also the formation process of the thrombus. And the results will be compared with in-vitro experiments, for example Ref. (8), in which the platelet deposition onto immobilized vWF or Fbg under flow was estimated, to verify our proposed modeling.

\section{Acknowledgments}

This research was supported by "Revolutionary Simulation Software (RSS21)" project supported by next-generation IT program of Ministry of Education, Culture, Sports, Science and Technology (MEXT), Grants in Aid for Scientific Research by the MEXT and JSPS Scientific Research in Priority Areas (768) "Biomechanics at Micro- and Nanoscale Levels", and Scientific Research(A) No.16200031 "Mechanism of the formation, destruction, and movement of thrombi responsible for ischemia of vital organs". Daisuke Mori acknowledges the support of the Tohoku University 21 COE Program "Future Medical Engineering Based on Bio-nanotechnology".

\section{References}

(1) Ruggeri, Z. M., Platelets in atherothrombosis, Nat Med, Vol.8, No.11 (2002), pp. 1227-1234.

(2) Rao, A. K., and Holmsen, H., Congenital disorders of platelet function, Semin Hematol, Vol.23, No.2 (1986), pp. 102-118.

(3) Takeoka, S., Teramura, Y., Ohkawa, H., Ikeda, Y., and Tsuchida, E., Conjugation of von Willebrand factor-binding domain of platelet glycoprotein $\mathrm{Ib}$ alpha to size-controlled albumin microspheres, Biomacromolecules, Vol.1, No.2 (2000), pp. 290-295.

(4) Lefkovits, J., Plow, E. F., and Topol, E. J., Platelet glycoprotein IIb/IIIa receptors in cardiovascular medicine, $N$ Engl J Med, Vol.332, No.23 (1995), pp. 1553-1559.

(5) Ruggeri, Z. M., Platelet interactions with vessel wall components during thrombogenesis, Blood Cells Mol Dis, Vol.36, No.2 (2006), pp. 145-147.

(6) Ruggeri, Z. M., 1998, Von Willebrand Factor and the Mechanisms of Platelet Functions, Springer-Verlag.

(7) Goto, S., Salomon, D. R., Ikeda, Y., and Ruggeri, Z. M., Characterization of the unique mechanism mediating the shear-dependent binding of soluble von Willebrand factor to platelets, J Biol Chem, Vol.270, No.40 (1995), pp. 23352-23361.

(8) Savage, B., Saldivar, E., and Ruggeri, Z. M., Initiation of platelet adhesion by arrest onto fibrinogen or translocation on von Willebrand factor, Cell, Vol.84, No.2 (1996), pp. 289-297.

(9) Sorensen, E. N., Burgreen, G. W., Wagner, W. R., and Antaki, J. F., Computational simulation of platelet deposition and activation: II. Results for Poiseuille flow over collagen, Ann Biomed Eng, Vol.27, No.4 (1999), pp. 449-458.

(10) Sorensen, E. N., Burgreen, G. W., Wagner, W. R., and Antaki, J. F., Computational simulation of platelet deposition and activation: I. Model development and properties, 
Ann Biomed Eng, Vol.27, No.4 (1999), pp. 436-448.

(11) Kamada, H., Tsubota, K., Wada, S., and Yamaguchi, T., Computer simulation of formation and collapse of primary thrombus due to platelet aggregation using particle method, Transactions of the Japan Society of Mechanical Engineers, Series B, Vol.72, No.717 (2006), pp. 1109-1115.

(12) Satoh, A., Chantrell, R. W., Coverdale, G. N., and Kamiyama, S., Stokesian Dynamics Simulations of Ferromagnetic Colloidal Dispersions in a Simple Shear Flow, $J$ Colloid Interface Sci, Vol.203, No.2 (1998), pp. 233-248.

(13) Satoh, A., Comparison of approximations between additivity of velocities and additivity of forces for Stokesian dynamics methods, J Colloid Interface Sci, Vol.243, No.2 (2001), pp. 342-350.

(14) Durlofsky, L., Brady, J. F., and Bossis, G., Dynamic Simulation of Hydrodynamically Interacting Particles, J Fluid Mech, Vol.180, (1987), pp. 21-49.

(15) Frojmovic, M. M., and Milton, J. G., Human platelet size, shape, and related functions in health and disease, Physiol Rev, Vol.62, No.1 (1982), pp. 185-261.

(16) Kim, S., and Karrila, S. J., 1991, Microhydrodynamics: Principles and Selected Applications, Butterworth-Heinemann, Stoneham.

(17) Hakuno, M., and Meguro, K., Simulation of Concrete-Frame Collapse Due to Dynamic Loading, Journal of Engineering Mechanics-Asce, Vol.119, No.9 (1993), pp. 1709-1723.

(18) Suzuki, H., Yamamoto, N., Tanoue, K., and Yamazaki, H., Glycoprotein Ib distribution on the surface of platelets in resting and activation states: an electron microscope study, Histochem J, Vol.19, No.3 (1987), pp. 125-136. 